\title{
Application of hyperspectral imaging to detect toxigenic Fusarium infection on cornmeal
}

\author{
V. PARRAG ${ }^{1,6 *}$ (D), Z. GILLAY ${ }^{1}$, Z. KOVÁCS ${ }^{1}$, A. ZITEK $^{2}$, K. BÖHM ${ }^{3}$, \\ B. HINTERSTOISSER ${ }^{3}$, R. KRSKA ${ }^{2,4,5}$, M. SULYOK ${ }^{2,4}$, J. FELFÖLDI $^{1}$, \\ F. FIRTHA ${ }^{1}$ and L. BARANYAI ${ }^{1}$
}

${ }^{1}$ Department of Physics and Control, Faculty of Food Science, Szent István University, Somlói u. 14-16, H-1118 Budapest, Hungary

${ }^{2}$ FFoQSI - Austrian Competence Centre for Feed and Food Quality, Safety \& Innovation, Technopark 1C, 3430 Tulln, Austria

${ }^{3}$ Institute of Physics and Materials Science, University of Natural Resources and Life Sciences, PeterJordan-Straße 82, 1190 Vienna, Austria

${ }^{4}$ Department of Agrobiotechnology, Institute of Bioanalytics and Agro-Metabolomics, IFA-Tulln, University of Natural Resources and Life Sciences, Konrad-Lorenz-Straße 20, 3430 Tulln an der Donau, Austria

${ }^{5}$ Institute for Global Food Security, School of Biological Sciences, Queens University Belfast, University Road, Belfast, BT7 1NN, United Kingdom

${ }^{6}$ Campden BRI Hungary, Haller u. 2, H-1096 Budapest, Hungary

\section{ORIGINAL RESEARCH PAPER}

Received: August 11, 2020 • Accepted: November 13, 2020

Published online: December 08, 2020

(C) 2020 The Author(s)

\section{ABSTRACT}

One of the most important food safety issues is the detection of mycotoxins, the ubiquitous, natural contaminants in cereals. Hyperspectral imaging (HSI) is a new method in food science, it can be used to predict non-destructively the changes in composition and distribution of compounds. That is why, in the last decade, the potential of HSI has been evaluated in many fields of food science, including mycotoxin research.

The aim of the recent study was to test the feasibility of HSI for the differentiation according to the toxin content of cornmeal samples inoculated with Fusarium graminearum, Fusarium verticillioides and Fusarium culmorum and samples with natural levels of mycotoxins. Samples were measured in the near

\footnotetext{
*Corresponding author. E-mail: v.parrag@campdenkht.com
} 
infrared wavelength range of $900-1,700 \mathrm{~nm}$ and mean spectra of selected regions of interest of each image were pre-treated using Savitzky-Golay smoothing and standard normal variate (SNV) method. On the spectra, partial least squares discriminant analysis (PLS-DA) was carried out according to the level of contamination. Partial least squares regression (PLSR) method was used to predict deoxynivalenol (DON) content of samples and the cumulative toxin content: the sum of fumonisins $\left(\mathrm{FB}_{1}, \mathrm{FB}_{2}\right)$ and $\mathrm{DON}$ content of samples. Based on the promising results of the study, HSI has the potential to be used as a preliminary testing method for mycotoxin content in feed materials.

\section{KEYWORDS}

cornmeal, hyperspectral imaging, HSI, mycotoxin, Fusarium

\section{INTRODUCTION}

The detection of fungal infections is of great importance in food science since one of the most serious problems of food safety is the presence of mycotoxins produced by microscopic fungi. Molds can grow on many kinds of substrates under different conditions therefore mycotoxins are unavoidable, ubiquitous natural contaminants of a wide range of food and feed, especially cereals.

Maize is produced in high amounts as food and feed and is consumed all around the world, the production of maize was in 2018 more than $10^{9}$ tons in the world and almost $7 \cdot 10^{7}$ tons in the countries of the European Union (FAOSTAT). It plays an important role in the human diet among others due to its high carbohydrate, especially starch content which builds $72-73 \%$ of the kernel weight (Food and Agriculture Organization of the United Nations, 1992).

Corn can be attacked by many types of molds producing toxigenic secondary metabolites (mycotoxins) such as aflatoxins, ochratoxin A, fumonisins, zearalenone, Type A and B trichothecenes like deoxynivalenol (Prieto-Simón et al., 2007).

Fusarium species are prevalent pathogens of maize, capable of producing several hundred compounds that have been described as toxic or potentially toxic secondary metabolites (Moretti, 2017), amongst others fumonisins and deoxynivalenol (DON), whose compounds cause esophageal cancer, throat irritation, gastrointestinal and abdominal pains, nausea, vomiting, diarrhea, hemorrhages, dizziness, fever and headache in humans (Prieto-Simón et al., 2007).

Trichothecenes such as deoxynivalenol are the most often associated mycotoxins of Fusarium with toxicoses in humans and livestock, second is the group of fumonisins (Moretti, 2017).

The trichothecenes are stable at temperatures of the processing steps of food and feed, therefore, this group of mycotoxins causes food safety risk even in processed food (Rocha et al., 2005).

In the countries of the European Union maximum levels for certain contaminants in foodstuffs as regards Fusarium toxins in maize and maize products are set by Commission Regulation (EC) No 1126/2007 of 28 September 2007 amending Regulation (EC) No 1881/2006. According to the regulation of the European Union maximum levels of mycotoxins regarding ground maize, not used for direct human consumption is in the case of deoxynivalenol $750 \mu \mathrm{g} /$ $\mathrm{kg}$, in the case of fumonisins (sum of $\mathrm{B}_{1}$ and $\mathrm{B}_{2}$ ) $1,400 \mu \mathrm{g} / \mathrm{kg}$ (Regulation, 2007).

Since the commonly used analytical methods to determine mycotoxins, such as enzyme-linked immunosorbent assays (ELISA) and LC-MS/MS are costly, time-consuming, and cannot be applied in the field, new methods appeared to detect mycotoxins and fungal infections recently (McMullin et al., 2014). 
IR spectroscopic approaches are an emerging field of food research as these techniques are rapid, non-destructive and easy to apply even in high throughput.

However, the currently used near infrared (NIR) based methods - like the applied hyperspectral imaging (HSI) - cannot determine mycotoxins in food samples directly due to lack of sensitivity. Instead, the alterations of spectral characteristics of crops upon fungal infections can be assessed. For instance, Fusarium species cause alterations in the carbohydrate and protein content (He \& Sun, 2015).

Studies dealing with the application of HSI in microbiology have increased rapidly in the past decade and have been reviewed by Gowen et al. (2015). HSI provides information about chemical changes in the sample, therefore, it has the potential to be used as a tool to gain fundamental understanding of microbiological processes (Gowen et al., 2015).

In the last few years, the number of papers dealing with the potential of hyperspectral imaging in IR for the detection of fungal infection and the presence of toxins in cereals is increasing.

HSI seems to be a promising method for detecting plant stress and disease, there has been a significant increase in scientific literature focusing on plant stress detection using hyperspectral image analysis in recent years (Lowe et al., 2017). Xing et al. provide an overview of the recent literature on the application of hyperspectral imaging for the rapid detection and sorting of mycotoxins and toxigenic fungi in food products (Xing et al., 2019). Imaging technics, spectroscopy, and hyperspectral imaging have been reviewed for the determination of contamination caused by toxigenic fungi in nuts and dried fruits with regard to aflatoxins (Wu et al. 2018). Studies have been also published about the detection of ochratoxin A contamination in stored wheat (Senthilkumar et al., 2016; 2017), barley (Senthilkumar et al., 2016), and about the detection of Fusarium caused damage on wheat (Barbedo et al., 2015; Bauriegel et al., 2011) and Italian durum wheat kernels (Serranti et al., 2013), as well as about the feasibility of HSI to detect DON content and Fusarium damage on single oat kernels (Tekle et al., 2015).

Del Fiore et al. investigated the potential of hyperspectral imaging technique in the wavelength range of 400-1,000 $\mathrm{nm}$ to detect different typical fungal infections of maize using Aspergillus, Fusarium and Penicillium strains for inoculation. Uninfected maize kernel samples from artificially infected samples could be discriminated only after 48 hours of inoculation with A. flavus and A. niger (del Fiore et al., 2010).

Barbedo et al. invented a DON screening algorithm using a few selected bands, which can classify Fusarium infected wheat kernels with 72 and $81 \%$ accuracies to three and two classes, respectively. They found that the direct estimation of DON content was unfeasible, however, an indirect method taking into account the correlation between Fusarium damage and DON content can be accurate enough to improve the process of DON screening (Barbedo et al., 2017).

Earlier experiments showed that fungal infection of Fusarium sp. is detectable using HSI in NIR region. The aim of this research was to evaluate the applicability of this method in this practical situation and to explore the boundaries of the usability of this technique.

\section{SAMPLES AND SAMPLE PREPARATION}

\section{Sample preparation}

Two hybrids of Zea mays were examined in this experiment. One hybrid (SBL) from Saatbau Linz (Linz Austria) is rather resistant to Fusarium infection, the other examined hybrid from Cereal Research Institute in Szeged, Hungary (CRC) is very susceptible to Fusarium. 
Since the presence of a low level of fungal toxin in maize is unavoidable, samples were not disinfected.

The control group of maize samples was not artificially infected and exhibited natural levels of toxin. Two other groups were infected via injection of the spores through silk with Fusarium graminearum and Fusarium verticillioides, respectively. One group of samples was treated with Fusarium culmorum by dipping a toothpick into the spore suspension and afterwards picking the maize ear with this toothpick.

\section{Determination of mycotoxin content}

Toxin content of samples by means of deoxynivalenol, fumonisins $-\mathrm{B}_{1}$ and $\mathrm{B}_{2}-$ and total toxin content was measured using an LC-MS/MS-based method (Malachová et al., 2014). In each group, different levels of total toxin content were found (Table 1).

\section{Hyperspectral imaging}

Samples were put in Petri dishes with a diameter of $5 \mathrm{~cm}$ and pressed before the measurements to reduce scattering effects. In each group, 8 repetitions were measured ( 8 Petri dishes were prepared - the total number of the prepared samples was 176).

Hyperspectral measurements were recorded using a pushbroom HSI instrument (Zeutec GmbH., Rendsburg, Germany, equipped with Xeva-USB-FPA-1.7-320-TE1 camera with InGaAs sensor $(12$ bit) $320 \times 256$ image size, ImSpector N17E spectrograph $5 \mathrm{~nm}$ spectral resolution) in the wavelength range of $900-1,700 \mathrm{~nm}$. The spatial resolution of the optics was adjusted to $0.172 \mathrm{~mm} / \mathrm{px}$. The image processing system and the sensor were controlled by Argus software (Firtha, 2011).

Table 1. Deoxynivalenol (DON), fumonisin $\mathrm{B}_{1}\left(\mathrm{FB}_{1}\right)$ and $\mathrm{B}_{2}\left(\mathrm{FB}_{2}\right)$ content of cornmeal samples (NI: not infected, G: infected with Fusarium graminearum, V: infected with Fusarium verticillioides, C: infected with Fusarium culmorum, SBL: hybrid from Saatbau Linz, Linz Austria; CRC: hybrid from Cereal Research Institute, Szeged, Hungary, LOD: limit of detection)

\begin{tabular}{|c|c|c|c|c|c|}
\hline Hybrid & Class & $\mathrm{DON}(\mathrm{mg} / \mathrm{kg})$ & $\mathrm{FB}_{1}(\mathrm{mg} / \mathrm{kg})$ & $\mathrm{FB}_{2}(\mathrm{mg} / \mathrm{kg})$ & Total $(\mathrm{mg} / \mathrm{kg})$ \\
\hline \multirow[t]{11}{*}{ SBL } & NI & 0.912 & 0.087 & 0.030 & 1.029 \\
\hline & NI & 0.096 & $<\mathrm{LOD}$ & $<\mathrm{LOD}$ & 0.096 \\
\hline & G & 32.160 & 0.001 & 0.000 & 32.161 \\
\hline & G & 19.040 & 0.586 & 0.163 & 19.789 \\
\hline & G & 5.632 & $<$ LOD & $<\mathrm{LOD}$ & 5.632 \\
\hline & V & 0.464 & 20.800 & 6.000 & 27.264 \\
\hline & V & 0.113 & 4.560 & 1.504 & 6.177 \\
\hline & $\mathrm{V}$ & 0.298 & 0.944 & 0.286 & 1.529 \\
\hline & $\mathrm{C}$ & 52.480 & 3.264 & 1.136 & 56.880 \\
\hline & $\mathrm{C}$ & 24.640 & 0.550 & 0.263 & 25.453 \\
\hline & C & 0.236 & 0.450 & 0.208 & 0.894 \\
\hline \multirow[t]{11}{*}{ CRC } & NI & 1.528 & 0.187 & 0.091 & 1.806 \\
\hline & NI & 0.215 & $<$ LOD & $<$ LOD & 0.215 \\
\hline & G & 50.160 & 0.832 & 0.262 & 51.254 \\
\hline & G & 30.320 & 0.041 & 0.020 & 30.381 \\
\hline & G & 14.640 & 0.067 & 0.027 & 14.734 \\
\hline & V & 0.723 & 52.320 & 18.400 & 71.443 \\
\hline & V & 1.680 & 22.240 & 7.616 & 31.536 \\
\hline & $\mathrm{V}$ & 0.734 & 7.688 & 2.280 & 10.702 \\
\hline & $\mathrm{C}$ & 73.840 & 2.728 & 1.032 & 77.600 \\
\hline & $\mathrm{C}$ & 20.800 & 2.992 & 0.976 & 24.768 \\
\hline & C & 1.184 & 1.448 & 0.669 & 3.301 \\
\hline
\end{tabular}


Before the measurement series, a two-point calibration was carried out using a diffuse reflectance standard containing rare earth elements (Sphereoptics).

Hyperspectral images were segmented, and noise reduction was carried out using MATLAB (2016. The MathWorks, Inc. Natick, Massachusetts, USA) software. On the hyperspectral images a $100 \times 100$ pixel, homogenously illuminated region was selected for further analysis. This region was split into 5 times $20 \times 100$ pixel regions, spectra of these regions of interest were afterwards averaged.

On the raw spectra, a Savitzky-Golay smoothing (2nd order polynomial, 25 points) and standard normal variate (SNV) transformation was carried out to decrease the unwanted effects of noise on the spectra (Wise et al., 2006).

Data analysis was carried out using RStudio version 0.99.896. (RStudio, Inc.). The toxin content of the samples was predicted with Partial least squares regression (PLSR) method (pls package, version 2.5-0) and the samples of different categories of contamination level were classified with partial least squares discriminant analysis (PLS-DA) method (mixOmics package, version 5.2.0).

Cumulated toxin content (sum of DON and fumonisins), the content of DON and fumonisins were predicted based on analytical results and hyperspectral data via PLSR. The $75 \%$ of the data were used for model building and $25 \%$ as the validation set.

For the regression, data were randomly selected according to the above-described ratio in each group for modeling and validation. This procedure was repeated 10 times and afterwards, the number of latent variables was increased by 5 to approximate the optimal numbers of latent variables. The same procedure was followed in case of classification with PLS-DA.

\section{RESULTS}

There are important differences in the metabolic activity of fungi to consider regarding the data analysis. In the case of the two studied hybrids, the average toxin content of more susceptible hybrid (CRC) groups were higher than the values of the more resistant one (SBL), corresponding to the expectations. Furthermore, there are general differences in toxin production of different Fusarium species: F. culmorum and F. graminearum produce trichothecenes, including deoxynivalenol (DON), while Fusarium verticilloides produces fumonisins (Jakucs and Vajna, 2003).

On the average reflectance spectra of sample groups (Fig. 1), no clear relationship between the reflectance values and classes could be detected, although the deviation in toxin content was significant (Table 1) and, therefore, the degree of infection might have been very different in one group.

The effect of water absorption was noticeable around 1,440-1,470 $\mathrm{nm}$ on the reflectance spectra (Fig. 1).

\section{Prediction of cumulated toxin content}

The best results regarding the root mean square error of validation (RMSEV) to predict the cumulated toxin content were achieved with 25 latent variables at $13.48 \mathrm{mg} / \mathrm{kg}$ while the highest value among the 10 repetitions was found at $15.12 \mathrm{mg} / \mathrm{kg}$. The average value of the coefficient of determination after 10 repetitions in the case of 25 latent variables was 0.974. Fig. 2 shows the prediction plot of PLSR regression of cumulated toxin content in the case of one validation 


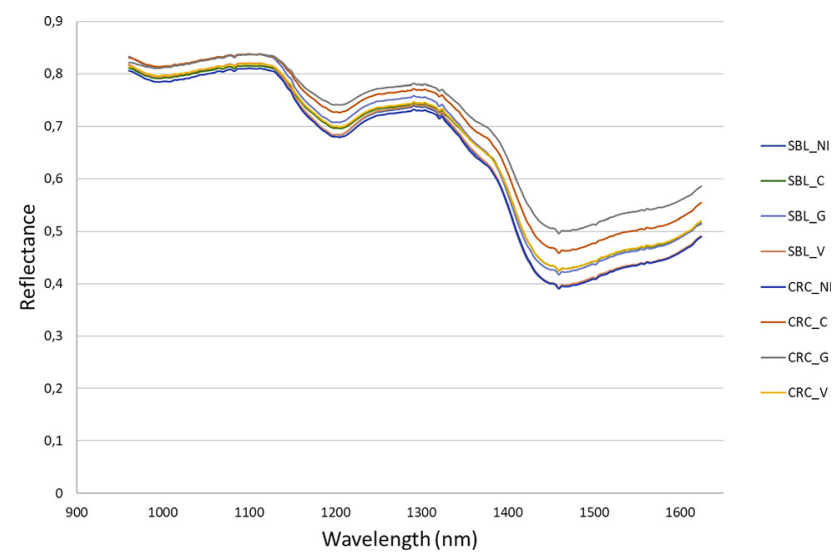

Fig. 1. Average reflectance spectra of sample groups samples (NI: not infected, G: infected with Fusarium graminearum, V: infected with Fusarium verticillioides, C: infected with Fusarium culmorum, SBL: hybrid from Saatbau Linz, Linz Austria; CRC: hybrid from Cereal Research Institute, Szeged, Hungary)

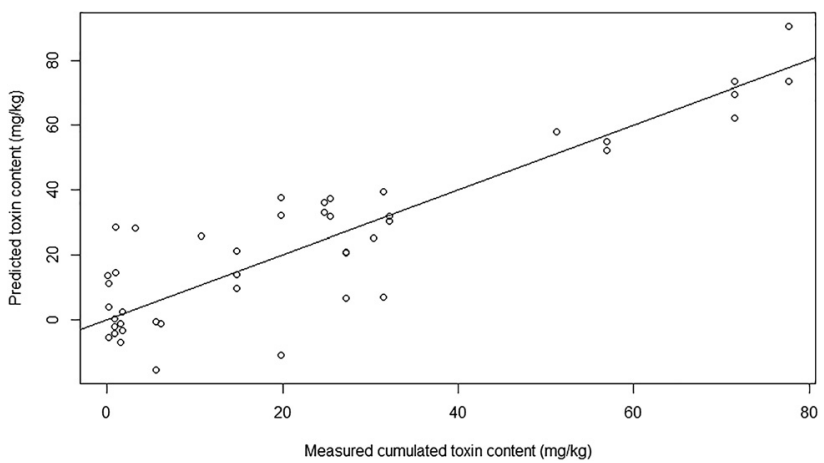

Fig. 2. Prediction plot of the PLSR regression of cumulated toxin content in case of one validation sample set

sample set. In the case of 25 latent variables the value of root mean square error of calibration (RMSEC) was $4.22 \mathrm{mg} / \mathrm{kg}$, whereas the value of RMSEV was $13.48 \mathrm{mg} / \mathrm{kg}$.

\section{Prediction of deoxynivalenol content}

The production of deoxynivalenol is characteristic in the case of F. culmorum and F. graminearum, therefore, the data of these species were used for the prediction.

The lowest average RMSEV value was achieved using 20 latent variables at $11.95 \mathrm{mg} / \mathrm{kg}$, while the average value of RMSE was $2.5 \mathrm{mg} / \mathrm{kg}$. On the prediction plot of the PLSR regression of DON toxin content in the case of one validation sample set (Fig. 3) the deviations from the predicted values were noticeable. The average value of the coefficient of determination for 10 repetitions, using 20 latent variables in the model building, was 0.985 . 


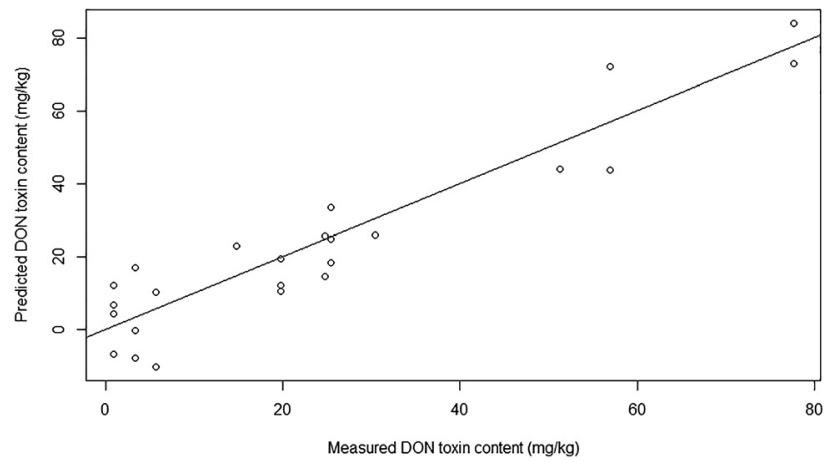

Fig. 3. Prediction plot of the PLSR regression of DON toxin content in case of one validation sample set

\section{Classification of groups according to mycotoxin contamination levels}

In the building of classification models of groups according to mycotoxin levels, the data of each group were used. Samples were categorized into three classes according to the cumulated toxin content (DON and fumonisins):

-high level of mycotoxin contamination: above $30 \mathrm{mg} / \mathrm{kg}$ (56 samples)

-medium level of mycotoxin contamination: between 30 and $15 \mathrm{mg} / \mathrm{kg}$ (32 samples)

-low level of mycotoxin contamination: below $15 \mathrm{mg} / \mathrm{kg}$ (88 samples).

Regarding the validation, the best result were achieved using 15 latent variables (Tables 2 and 3 ). In this case, the average ratio of the correctly classified samples after 10 repetitions achieved $98.8 \%$.

The lowest value of the ratio of correctly classified samples was given in the case of medium level of mycotoxin contamination (also the lowest number of samples is in this category). It was followed by the class of high level of mycotoxin content, and the group with low contamination level (Table 3).

\section{DISCUSSION}

According to the presented results, the examination of mycotoxin contamination in maize products proved to be possible through the application of a near infrared hyperspectral imaging system. However, the achieved accuracy was still relatively low, therefore, it should be applied preferably for a quick screening of feed samples. However, high throughput can be achieved, and

Table 2. Average of validation results of PLS-DA classification after 10 repetitions in case of different numbers of latent variables

Number of latent variables

Average of the ratio of correctly classified samples (\%) 
Table 3. Confusion matrix of validation of PLS-DA classification according to contamination level with 15 latent variables based on the average of 10 repetitions

\begin{tabular}{lccr}
\hline & \multicolumn{3}{c}{ Real class } \\
\cline { 2 - 4 } Predicted class & High & Medium & Low \\
\hline High & $99 \%$ & $1 \%$ & $0 \%$ \\
Medium & $0 \%$ & $98 \%$ & $0 \%$ \\
Low & $1 \%$ & $1 \%$ & $100 \%$ \\
\hline
\end{tabular}

an installation at site is possible. Our results are in accordance with the conclusion of $\mathrm{He}$ and Sun, (2015) - that the method cannot detect mycotoxins directly, but it provides information about the chemical changes of the samples due to the metabolic activity of microscopic fungi.

Using this method, a higher amount of maize products can be measured and with appropriate calibration, as preliminary testing method to screen suspicious samples it could complement the well established extensively applied analytical measurements, to support the improvement of feed safety along the production chain.

In the present study, the mycotoxin concentration of inoculated samples was significantly higher in most cases than the legal maximum limits defined by the commission regulation (EC) No $1126 / 2007$ setting maximum levels for certain contaminants in foodstuffs regarding Fusarium toxins in maize and maize products. Still, the experiments with these infected samples revealed the sensitivity and potential application of the method. The measurement could be continued with a higher amount of samples having mycotoxin levels below the legal maximum limit as well as the data could be extended with the determination of other regulated mycotoxins such as zearalenone. Moreover improved hyperspectral imaging systems with higher sensitivities and higher spectral resolutions like the Specim F17 have become available at much lower costs in the meantime.

\section{CONCLUSIONS}

Since mycotoxins are causing extremely serious food safety risk, the application of methods of high specificity is essential. The application of HSI as complementary method together with the commonly used and well established analytical techniques could enable the rapid inline measurement of feed material. Therefore, it could contribute to the improvement of feed safety along the production chain significantly. In future studies so far unexploited potentials of HSI with regard to the examination of other mycotoxin-producing micromycetes potentially occurring in feedstuff, and other mycotoxins e.g. zearalenone, should be assessed.

The results show that HSI was able to classify mycotoxin contamination levels in feed with $98.8 \%$ accuracy. Optimized HSI was able to reach $2.5 \mathrm{mg} / \mathrm{kg}$ accuracy in the quantification of toxins, but the significant difference between calibration and validation revealed the need for new measurements to build more robust models.

\section{ACKNOWLEDGEMENT}

We acknowledge the support of the project by the COMET-K1 competence center FFoQSI. The COMET-K1 competence center FFoQSI is funded by the Austrian ministries BMVIT, BMDW 
and the Austrian provinces Niederösterreich, Upper Austria and Vienna within the scope of COMET- Competence Centers for Excellent Technologies. The program COMET is handled by the Austrian Research Promotion Agency FFG. The authors also would like to thank the Austrian-Hungarian Action Foundation (Stiftung Aktion Österreich-Ungarn/AÖU/) for the support of the research with the Ernst Mach Scholarship.

\section{REFERENCES}

Barbedo, J.G.A., Tibola, C.S., and Fernandes, J. M. C. (2015). Detecting Fusarium head blight in wheat kernels using hyperspectral imaging. Biosystems Engineering, 131: 65-76.

Barbedo, J.G.A., Tibola, C.S., and Lima, M.I.P. (2017). Deoxynivalenol screening in wheat kernels using hyperspectral imaging. Biosystems Engineering, 155: 24-32.

Bauriegel, E., Giebel, A., Geyer, M., Schmidt, U., and Herppich, W.B. (2011). Early detection of Fusarium infection in wheat using hyper-spectral imaging. Computers and Electronics in Agriculture, 75(2): 304312.

del Fiore, A., Reverberi, M., Ricelli, A., Pinzari, F., Serranti, S., Fabbri, A.A., Bonifazi, G., and Fanelli, C. (2010). Early detection of toxigenic fungi on maize by hyperspectral imaging analysis. International Journal of Food Microbiology, 144(1): 64-71.

Firtha, F. (2011). Argus software.

Food and Agriculture Organization of the United Nations. (1992). Maize in human nutrition. Rome: Food and Agriculture Organization of the United Nations, (No.25), p. 160.

Gowen, A.A., Feng, Y., Gaston, E., and Valdramidis, V. (2015). Recent applications of hyperspectral imaging in microbiology. Talanta, 137: 43-54.

He, H.-J. and Sun, D.-W. (2015). Hyperspectral imaging technology for rapid detection of various microbial contaminants in agricultural and food products. Trends in Food Science \& Technology, 46(1): 99-109.

Jakucs, E. and Vajna, L. (Eds.). (2003). Mikológia. Agroinform, 514.

Lowe, A., Harrison, N., and French, A.P. (2017). Hyperspectral image analysis techniques for the detection and classification of the early onset of plant disease and stress. Plant Methods, 13(1): 80. BioMed Central Ltd.

MATLAB. (2016). Natick, MA: The MathWorks, Inc. (software).

Malachová, A., Sulyok, M., Beltrán, E., Berthiller, F., and Krska, R. (2014). Optimization and validation of a quantitative liquid chromatography-tandem mass spectrometric method covering 295 bacterial and fungal metabolites including all regulated mycotoxins in four model food matrices. Journal of Chromatography A, 1362: 145-156.

McMullin, D., Mizaikoff, B., and Krska, R. (2014). Advancements in IR spectroscopic approaches for the determination of fungal derived contaminations in food crops. Analytical and Bioanalytical Chemistry, 407(3): 653-660.

Moretti, A. (2017). Mycotoxigenic Fungi. mycotoxigenic fungi: methods and protocols, Methods in Molecular Biology, 1542.

Prieto-Simón, B., Noguer, T., and Campàs, M. (2007). Emerging biotools for assessment of mycotoxins in the past decade. TrAC Trends in Analytical Chemistry, 26(7): 689-702.

Regulation, (Ec) No 1126/2007 Commission. (2007). (EC) No 1126/2007 Commission Regulation (EC) No 1126/2007 of 28 September 2007 amending Regulation (EC) No 1881/2006 setting maximum levels for certain contaminants in foodstuffs as regards Fusarium toxins in maize and maize products. (EC) No 1126/2007 COMMISSION REGULATION, (1126), 4. 
Rocha, O., Ansari, K., and Doohan, F.M. (2005). Effects of trichothecene mycotoxins on eukaryotic cells: A review. Food Additives and Contaminants, 22(4): 369-378.

Senthilkumar, T., Jayas, D.S., White, N.D.G., Fields, P.G., and Gräfenhan, T. (2016). Detection of fungal infection and Ochratoxin A contamination in stored barley using near-infrared hyperspectral imaging. Biosystems Engineering, 147: 162-173.

Senthilkumar, T., Jayas, D.S., White, N.D.G., Fields, P.G., and Gräfenhan, T. (2017). Detection of ochratoxin A contamination in stored wheat using near-infrared hyperspectral imaging. Infrared Physics \& Technology, 81: 228-235.

Serranti, S., Cesare, D., and Bonifazi, G. (2013). The development of a hyperspectral imaging method for the detection of Fusarium-damaged, yellow berry and vitreous Italian durum wheat kernels. Biosystems Engineering, 115(1): 20-30.

Tekle, S., Mage, I., Segtnan, V.H., and Bjornstad, A. (2015). Near-infrared hyperspectral imaging of Fusarium-damaged oats (Avena sativa L.). Cereal Chemistry, 92(1): 73-80.

Wise, B.M., Gallagher, N.B., and Windig, W. (2006). Chemometrics Tutorial for PLS _ Toolbox and Solo. $\mathrm{Wu}, \mathrm{Q}$., Xie, L., and Xu, H. (2018). Determination of toxigenic fungi and aflatoxins in nuts and dried fruits using imaging and spectroscopic techniques. Food Chemistry, 252: 228-242.

Xing, F., Yao, H., Liu, Y., Dai, X., Brown, R. L., Bhatnagar, D. (2019). Recent developments and applications of hyperspectral imaging for rapid detection of mycotoxins and mycotoxigenic fungi in food products. Critical Reviews in Food Science and Nutrition, 59(1): 173-180.

Open Access. This is an open-access article distributed under the terms of the Creative Commons Attribution 4.0 International License (https://creativecommons.org/licenses/by/4.0/), which permits unrestricted use, distribution, and reproduction in any medium, provided the original author and source are credited, a link to the CC License is provided, and changes - if any - are indicated. (SID_1) 